\title{
Effectiveness of pulmonary rehabilitation in COPD with mild symptoms: a systematic review with meta-analyses
}

\author{
Mette Rugbjerg' \\ Ulrik Winning lepsen' \\ Karsten Juhl Jørgensen ${ }^{2}$ \\ Peter Lange ${ }^{1,3,4}$ \\ 'The Centre of Inflammation \\ and Metabolism and The Centre \\ for Physical Activity Research, \\ Department of Infectious Diseases, \\ Rigshospitalet, University of \\ Copenhagen, Copenhagen, Denmark; \\ ${ }^{2}$ The Nordic Cochrane Centre, \\ Rigshospitalet, Copenhagen, Denmark; \\ ${ }^{3}$ Department of Respiratory Medicine, \\ University Hospital Hvidovre, \\ Copenhagen, Denmark; ${ }^{4}$ Department \\ of Public Health, Section of Social \\ Medicine, University of Copenhagen, \\ Copenhagen, Denmark
}

This article was published in the following Dove Press journal: International Journal of COPD

17 April 2015

Number of times this article has been viewed

Purpose: Most guidelines recommend pulmonary rehabilitation (PR) for patients with chronic obstructive pulmonary disease (COPD) and modified Medical Research Council dyspnea scale (mMRC) levels $\geq 2$, but the effectiveness of PR in patients with less advanced disease is not well established. Our aim was to investigate the effects of PR in patients with COPD and mMRC $\leq 1$. Methods: The methodology was developed as a part of evidence-based guideline development and is in accordance with the principles of the Grading of Recommendations Assessment, Development and Evaluation (GRADE) Working Group. We identified randomized controlled trials (RCTs) through a systematic, multidatabase literature search and selected RCTs comparing the effects of PR with usual care in patients with COPD and $m M R C \leq 1$. Predefined critical outcomes were health-related quality of life (HRQoL), adverse effects and mortality, while walking distance, maximal exercise capacity, muscle strength, and dropouts were important outcomes. Two authors independently extracted data, assessed trial eligibility and risk of bias, and graded the evidence. Meta-analyses were performed when deemed feasible.

Results: Four RCTs (489 participants) were included. On the basis of moderate-quality evidence, we found a clinically and statistically significant improvement in short-term HRQoL of 4.2 units (95\% confidence interval [CI]: [ -4.51 to -3.89$]$ ) on St George's Respiratory Questionnaire, but not at the longest follow-up. We also found a statistically significant improvement of $25.71 \mathrm{~m}$ (95\% CI: [15.76-35.65]) in the 6-minute walk test with PR; however, this improvement was not considered clinically relevant. No difference was found for mortality, and insufficient data prohibited meta-analysis for muscle strength and maximal exercise capacity. No adverse effects were reported.

Conclusion: We found a moderate quality of evidence suggesting a small, significant improvement in short-term HRQoL and a clinically nonsignificant improvement in walking distance following PR in patients with COPD and mild symptoms. This resulted in a weak recommendation of routine PR in these patients using the GRADE approach.

Keywords: pulmonary disease, COPD, COPD with mild symptoms, physical activity, pulmonary rehabilitation, health-related quality of life

\section{Introduction}

The beneficial effects of pulmonary rehabilitation (PR) in patients with advancedstage chronic obstructive pulmonary disease (COPD) are well established. ${ }^{1}$ In most countries, PR is recommended for COPD patients with a dyspnea score of $\geq 2$ on the modified Medical Research Council dyspnea scale (mMRC). ${ }^{2-5}$ In these patients, PR results in increased health-related quality of life (HRQoL), increased exercise capacity, and decreased dyspnea. ${ }^{1}$ Patients with less advanced COPD and fewer symptoms are not routinely offered PR. 
It has been suggested that the lack of rehabilitation in patients with COPD and mild symptoms according to mMRC scoring (mMRC $\leq 1)$ may result in less focus on nonpharmacological strategies such as smoking cessation and may result in a faster deterioration in the patients' clinical status due to physical inactivity. ${ }^{4}$

By extrapolating known benefits of physical exercise in a healthy population to COPD patients with mild symptoms, it could be assumed that these patients also will benefit from physical activity. ${ }^{6}$ In population studies, reduced levels of physical activity in patients with all grades of COPD are associated with higher risk of hospital admissions and increased mortality; but it is difficult to determine whether reduced physical activity was causal or consequential to the deteriorating health status. ${ }^{7-9}$ However, a recent large cluster-randomized trial in a primary care setting by Kruis et $\mathrm{al}^{10}$ investigated the effects of integrated COPD management (including exercise) in patients with mean MRC levels of 2 and found no significant effects on the quality of life, hospital admissions, and hospital days.

In this systematic review, we used the Grading of Recommendations Assessment, Development and Evaluation (GRADE) Working Group tool to substantiate and formulate a recommendation on PR in less symptomatic patients with COPD $(\mathrm{mMRC} \leq 1)$. The study was initiated by the Danish Health and Medicines Authority in the context of a larger project to produce evidence-based national clinical guidelines. ${ }^{11}$

\section{Methods}

\section{Protocol and registration}

We did not publish a protocol of prespecified objectives and methods since this review was performed according to standardized methods within the Danish Health and Medicines Authority using GRADE guidelines for the process. ${ }^{12}$ Further, the population, intervention, control intervention, as well as critical and important outcomes (PICO) were decided by the working-group members prior to our literature search. ${ }^{13}$

\section{Eligibility criteria}

Randomized controlled trials (RCTs) that compared the effects of PR with usual care in patients with COPD and mild symptoms according to dyspnea scores, $\mathrm{mMRC} \leq 1$, were considered for inclusion, as were systematic reviews and guidelines based on RCTs. Studies were excluded if they were not RCTs or did not cover the predefined PICO. Our prespecified outcomes were evaluated immediately after PR or at the longest follow-up. The critical outcomes included HRQoL, adverse effects, and mortality, while important outcomes were walking distance (6-minute walk test [6MWT] or shuttle walk test), maximal exercise capacity, muscle strength, and dropout rates. We included manuscripts in English, Danish, Swedish, and Norwegian.

\section{Information sources}

Trials were identified searching multiple electronic databases, including Medline, Embase, CINAHL, PsycINFO, G-I-N International, NICE, National Guideline Clearinghouse, Scottish Intercollegiate Guidelines, HTA (The Health Technology Assessment) Database, The Cochrane Library, SBU (Sweden), Socialstyrelsen (Sweden), Helsedirektoratet (Norway), Kunnskapssenteret (Norway), PEDro and OTseeker. The last search was performed on 20 November, 2013.

\section{Search}

A research librarian performed the literature search in cooperation with UWI as part of a comprehensive guideline development within the Danish Health and Medicines Authority. First, we performed a broad multidatabase search yielding a total of 2,412 records, followed by a second more specific search for RCTs in Embase and Medline, where 95 records were identified. All records were screened for relevant titles and abstracts, while reference lists of included studies were assessed for further eligible literature (UWI). We searched for guidelines and systematic reviews within the last 10 years, but no specific time restrains were applied to the second search. The specific search strategies are shown in Supplementary materials - Section A.

\section{Study selection}

The full texts of relevant guidelines ${ }^{2,3}$ were evaluated by two authors independently using the AGREE II (Appraisal of Guidelines for Research and Evaluation) tool (Supplementary materials - Sections B and C), while eligible systematic reviews were assessed using AMSTAR (A Measurement Tool to Assess Systematic Reviews) (Supplementary materials - Section D). On the basis of the AGREE II and AMSTAR assessments, we determined whether the guidelines and reviews could be used directly or in part (eg, literature, quality assessment, and/or meta-analyses) in our study. The AGREE II assessments were also used for other related PICOs within the Danish guideline. RCTs were included if they met the prespecified PICO criteria and were not already included through guidelines or reviews. Any disagreements between reviewers were resolved through discussion.

\section{Data collection process}

Data extraction was performed independently by two authors in a predefined Microsoft Excel 2010 spreadsheet 
(KJJ, UWI) standardized for guideline development within the Danish Health and Medicines Authority, and any disagreements between the authors were solved though discussion. In addition, contact with three corresponding authors of the included RCTs was established (MR) to clarify study design and access additional data. In order to estimate the effect of PR on HRQoL using St George's Respiratory Questionnaire (SGRQ), both directly after the intervention and at the longest follow-up, additional data were provided from Gottlieb et a ${ }^{14}$ and van Wetering et al. ${ }^{15}$ The SGRQ scale ranges from 0 to 100 units, and changes of $>4$ are regarded clinically relevant. ${ }^{16}$ Additional data regarding the 6MWT were provided by both van Wetering et $\mathrm{al}^{15}$ and Román et al. ${ }^{17}$ Regarding the 6MWT, the minimal clinically important difference is $54 \mathrm{~m} .{ }^{18}$

\section{Risk of bias in individual studies}

The risk of bias in each study was assessed using the Cochrane Risk of Bias Tool ${ }^{19}$ by two independent authors (KJJ, UWI). Disagreements were solved through discussion.

\section{Summary measures}

All outcomes were assessed using random-effects metaanalyses. Effects of dichotomous outcomes were presented using odds ratios (ORs), and continuous outcomes were presented using mean differences if measured on the same scale, while standardized mean differences were used if different scales measuring the same outcome needed to be combined. Review Manager version 5.3 was used for statistical analyses. ${ }^{20}$

\section{Synthesis of results}

An $I^{2}$ value of less than $40 \%$ was regarded to indicate acceptable heterogeneity. Statistical significance was assessed using 95\% confidence intervals (CIs).

\section{Risk of bias for individual outcomes across studies}

Two authors (UWI, KJJ) independently evaluated the strength of the evidence for each outcome across studies using the GRADE criteria, and any disagreements were resolved through discussion. If our confidence in the effect measure was downgraded, the reasons were mentioned in footnotes to the "summary of findings" table (Table 1). Our overall confidence of the evidence for the intervention was determined by the critical outcome with the lowest rating. ${ }^{21}$

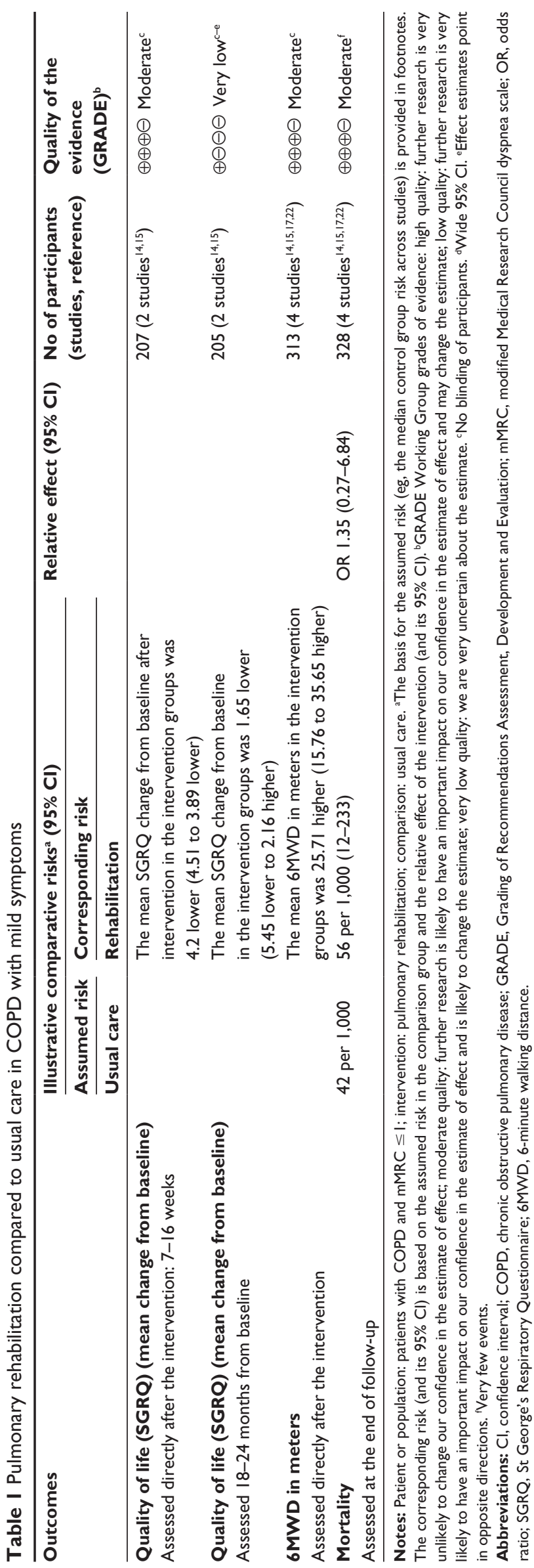




\section{Results}

\section{Study selection}

Two national guidelines, ${ }^{2,3}$ one systematic review, ${ }^{21}$ and four RCTs ${ }^{14,15,17,22}$ were included. Neither the guidelines nor the systematic review reported any overall effect estimates and contributed with reference lists only. Data from the four RCTs were included in our meta-analyses. ${ }^{14,15,17,22}$ A flow diagram of the selection process is presented in Figure 1.

\section{Characteristics of included studies}

The four eligible RCTs included a total of 489 participants with mean ages ranging from 61.3 to 74.1 years. Three of the four studies reported a male predominance (Table 2). ${ }^{15,17,22}$ The PR exercise programs carried out by the intervention groups varied in duration between 7 weeks ${ }^{14}$ and 6 months, ${ }^{22}$ frequencies of two to three times weekly, and exercise durations of 30-90 minutes followed by maintenance programs of various lengths.
Breathing techniques, respiratory physiotherapy, and educational programs in physiology and anatomy were additional elements in combination with the exercise training in the four studies. ${ }^{14,15,17,22}$ All the four studies were performed in an outpatient setting, but one study also included exercises at home. ${ }^{15}$ The control groups received usual care consisting of medical treatment, while one study also offered smoking cessation advice. ${ }^{15}$ No differences in baseline characteristics were reported in any of the four studies. The characteristics of the included studies are summarized in detail in Table 2.

\section{Risk of bias within studies}

The allocation concealment was not described in two studies, ${ }^{15,22}$ while one study did not report the randomization process. ${ }^{14}$ None of the studies blinded the participants or personnel, as this was impossible due to the intervention. Owing to high dropout rates in three of the studies, ranging from $20.6 \%$ to $55.7 \%$, a high risk of attrition bias was estimated (Table 3). ${ }^{14,15,17}$ No selective reporting of outcomes or

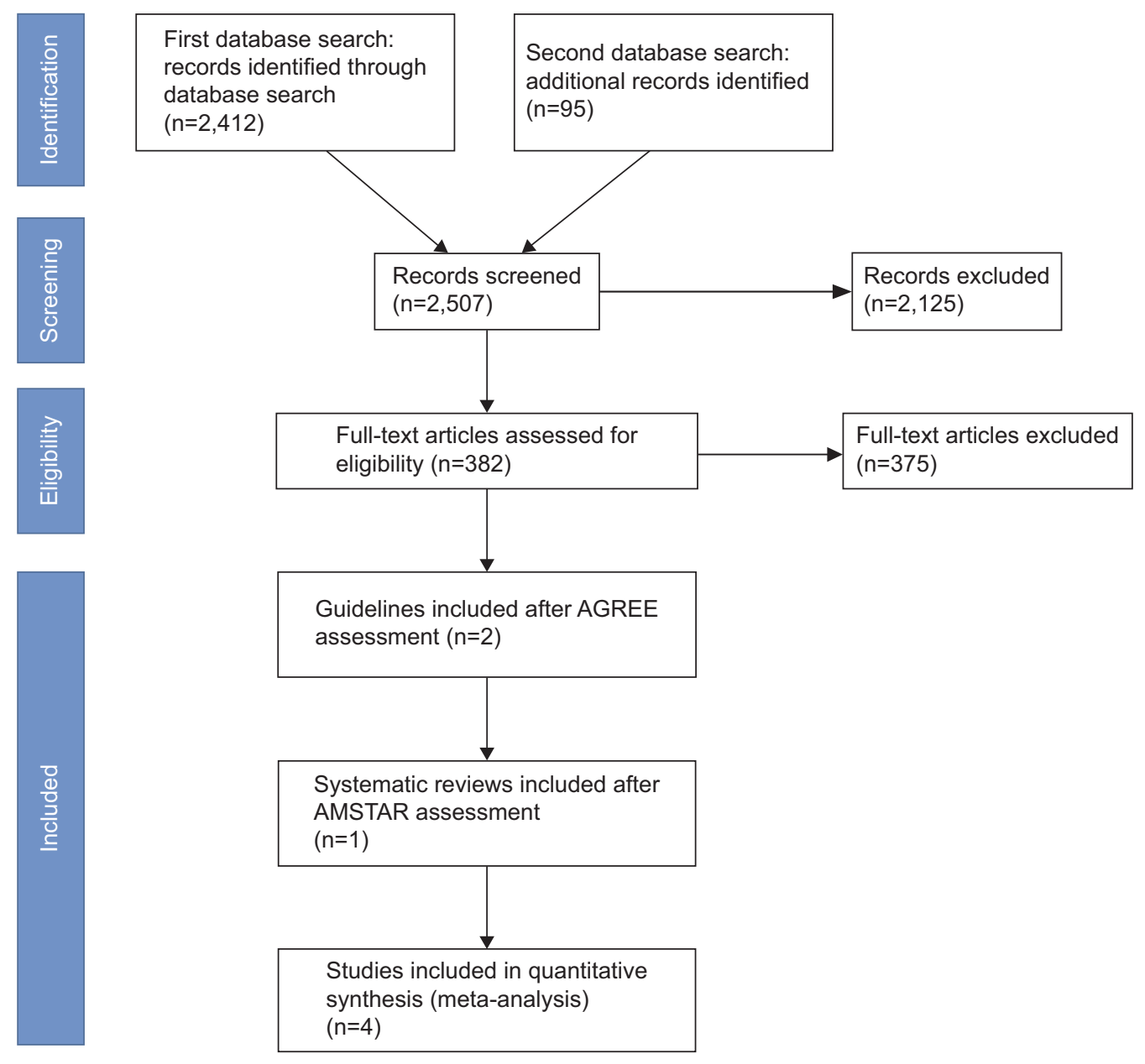

Figure I Flow diagram showing the selection process of material from identification to inclusion. Abbreviations: AGREE, Appraisal of Guidelines for Research and Evaluation; AMSTAR, A Measurement Tool to Assess Systematic Reviews. 


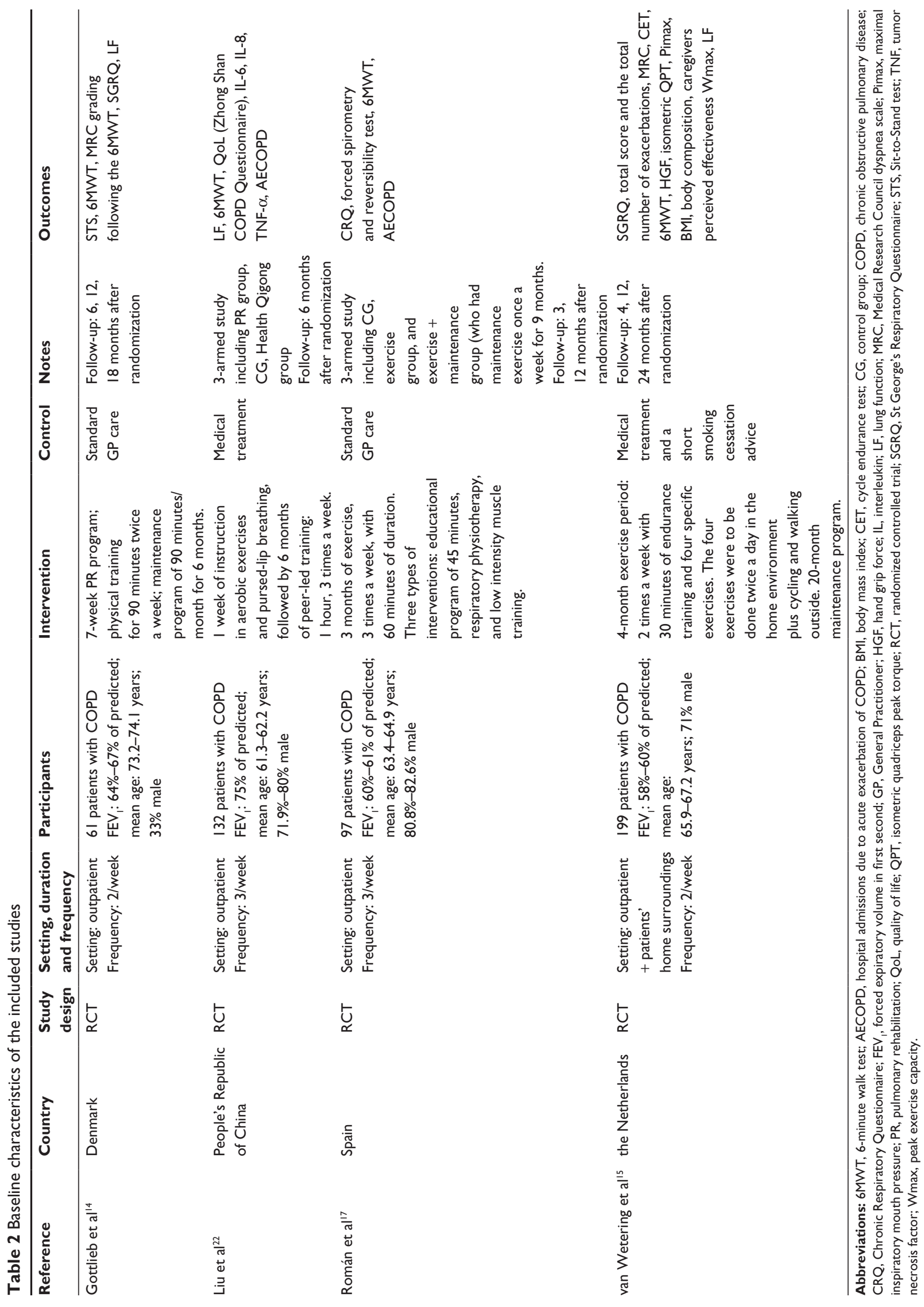




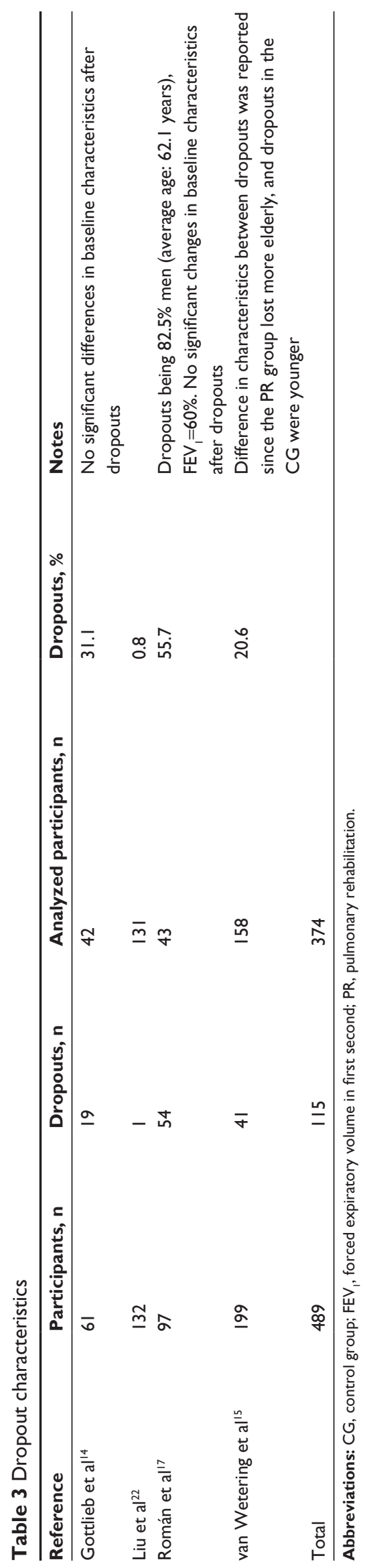

other sources of bias were detected. The risk of bias table is shown in Figure 2.

\section{Effects of the intervention}

A meta-analysis could be performed for four predefined outcomes.

\section{Health-related quality of life}

The HRQoL was assessed using SGRQ. Two studies were included, ${ }^{14,15}$ and data from 207 participants were pooled in a meta-analysis evaluating HRQoL directly after the PR and showed a small but statistically and clinically significant improvement of 4.2 units on the SGRQ scale (0-100 points) (95\% CI: [ -4.51 to -3.89$]$ ) in the PR group, compared with the control group (Figure 3), and there was no observed heterogeneity $\left(I^{2}=0 \%\right)$. Since the participants were not blinded, ${ }^{14,15}$ the quality of evidence for the short-term evaluation of HRQoL was downgraded to moderate (Table 1).

The same two studies ${ }^{14,15}$ also provided data from 205 participants on the effects of PR on HRQoL 18-24 months from baseline, and showed no statistically or clinically relevant difference (mean difference: $-1.65 ; 95 \%$ CI: $[-5.45$ to 2.16$]$ ) and an $I^{2}$ value of $25 \%$ (Figure 4 ).

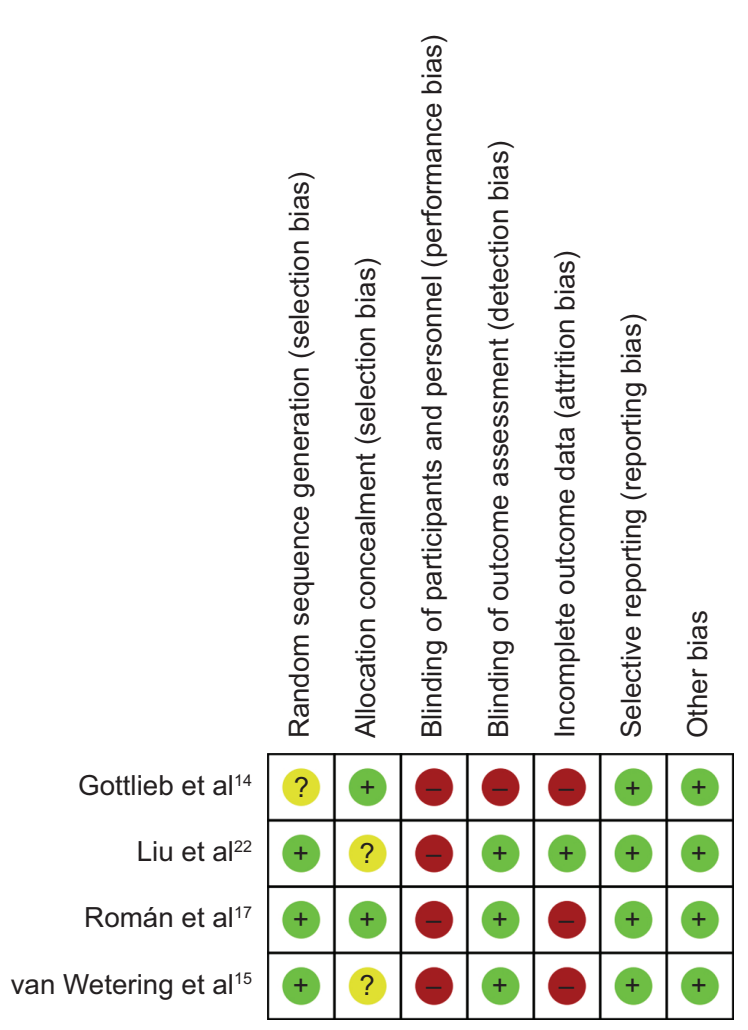

Figure 2 Risk of bias summary: the review authors' evaluations of each risk of bias item for each included study.

Notes: Green, low risk of bias; yellow, unclear risk of bias; red, high risk of bias. 


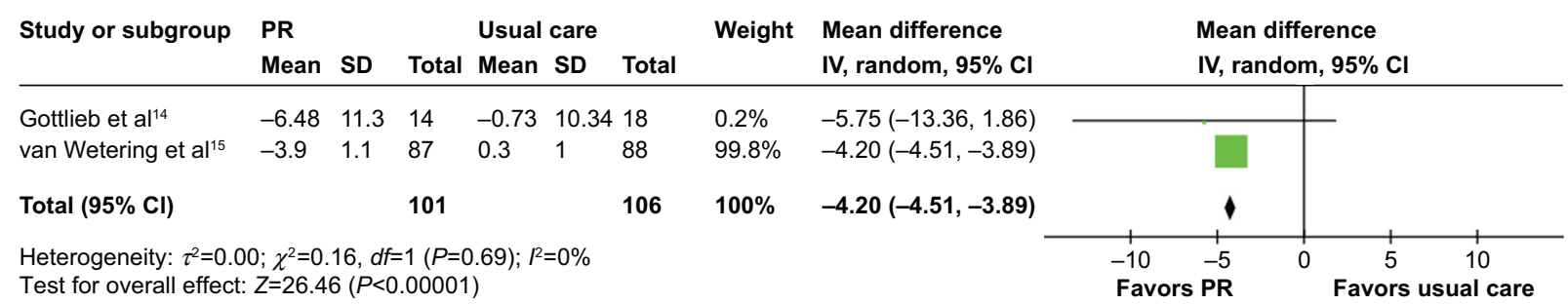

Figure 3 PR versus usual care: short-term evaluation of health-related quality of life measured by St George's Respiratory Questionnaire.

Abbreviations: $\mathrm{Cl}$, confidence interval; $d f$, degrees of freedom; IV, independent variable; PR, pulmonary rehabilitation; SD, standard deviation; $匚$, point estimate; $\$$, pooled effect estimate.

Our confidence in this result was downgraded to very low due to lack of blinding, dropouts, a wide CI, and effect estimates for the individual trials pointing in opposite directions, reflecting inconsistency (Table 1).

\section{Adverse effects}

None of the included studies reported on adverse effects from PR. . $^{4,15,17,22}$

\section{Walking distance}

The 6-minute walking distance (6MWD) was investigated in all four studies. Pooling the results of 157 and 156 participants from usual care and PR, respectively, ${ }^{14,15,17,22}$ yielded a statistically significant mean difference in walking distance of $25.71 \mathrm{~m}$, favoring PR (95\% CI: [15.76-35.65]). No heterogeneity was found, $I^{2}=0 \%$ (Figure 5 ). Lack of blinding of the participants led to downgrading of the confidence in our effect estimate to moderate (Table 1).

\section{Mortality}

Total mortality was reported in the four studies, including 328 randomized participants with 165 and 163 participants in the usual care group and PR group, respectively. ${ }^{14,15,17,22}$ Nine events were reported in the PR group, whereas seven events were reported in the control group. No statistically significant difference was found in our analysis, $\mathrm{OR}=1.35$
(95\% CI: [0.27-6.84]) (Figure 6), but the CI was wide due to the small number of events. Accordingly, our confidence in the effect estimate was downgraded to moderate due to imprecision (Table 1).

\section{Muscle strength}

The strength of the quadriceps muscle was assessed in only one study, with 87 and 88 participants in the PR and usual care group, respectively. No significant difference was found..$^{15}$ The same study investigated hand grip force and found a statistically significant improvement in the PR group (mean [standard error] change from baseline was 2.9 (1.1) Ib in the PR group, compared to $-1.2(1.2) \mathrm{Ib}$ in the control group, $P<0.01$ ), suggesting a beneficial effect of $P R$ on hand grip force, although this result was found in only one study. The quality of evidence was downgraded due to the lack of blinding and inclusion of only one study.

\section{Maximal exercise capacity}

One study investigated the effects of PR on maximal exercise capacity and stated that PR led to an improvement of $234 \mathrm{sec}-$ onds in a cycling endurance test $(P=0.04),{ }^{15}$ an improvement that was regarded as clinically relevant. ${ }^{23}$ The corresponding improvement in the control group was 29 seconds. ${ }^{15}$ Lack of blinding and lack of data led to downgrading the quality of the evidence to low.

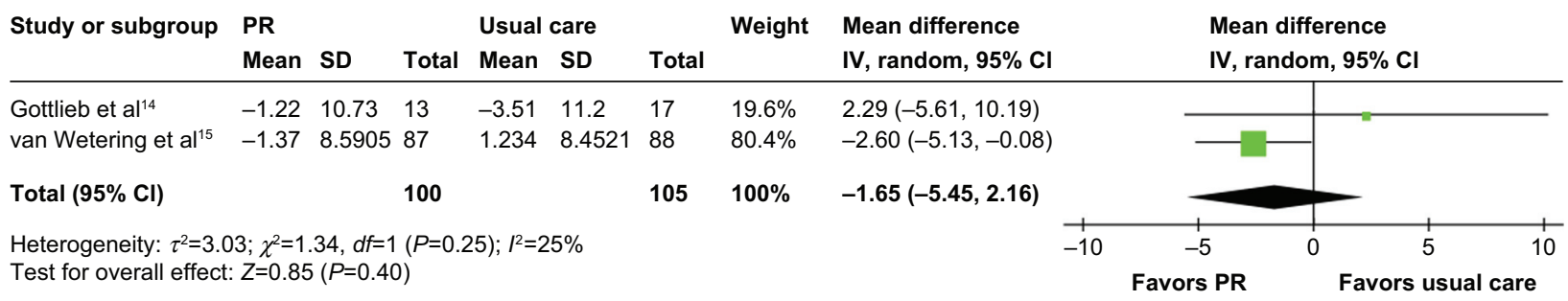

Figure 4 PR versus usual care: evaluation of health-related quality of life measured on St George's Respiratory Questionnaire at the longest follow-up (I8-24 months from baseline).

Abbreviations: $\mathrm{Cl}$, confidence interval; $d f$, degrees of freedom; IV, independent variable; PR, pulmonary rehabilitation; SD, standard deviation; $\square$, point estimate; $\$$, pooled effect estimate. 


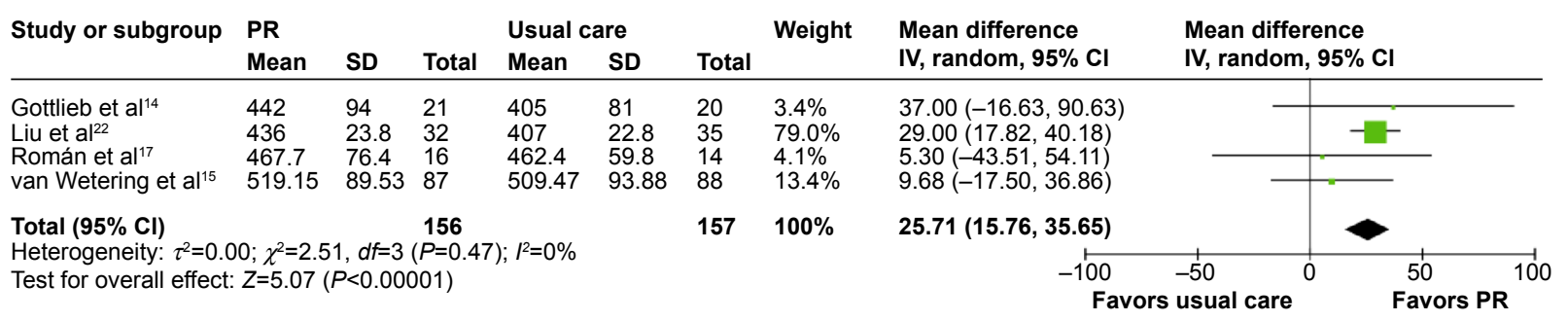

Figure 5 PR versus usual care: walking distance (6-minute walking distance).

Abbreviations: $\mathrm{Cl}$, confidence interval; df, degrees of freedom; IV, independent variable; PR, pulmonary rehabilitation; SD, standard deviation; effect estimate.

\section{Dropouts}

The dropouts reported in the four studies varied from 1 to 54 , ranging from $0.8 \%$ to $55.7 \%$ of the randomized participants (Table 3). ${ }^{14,15,17,22}$

\section{Discussion}

\section{Summary of the evidence}

The results of the meta-analyses suggested small but statistically and clinically significant improvements of HRQoL in patients with COPD and mild symptoms immediately after participation. However, no long-lasting benefits were found 18-24 months from baseline. Walking distance also improved, but the effect was too small to be considered clinically relevant. In accordance with the GRADE Working Group recommendations, we deemed the overall quality of the evidence to be "very low", reflecting the lowest quality rating of our critical outcomes.

\section{Our findings in relation to other studies}

Jácome and Marques ${ }^{21}$ presented a systematic review which similarly indicated that PR may be beneficial to patients with COPD and mild symptoms. However, this study differs methodologically from our study, given that the search strategy presented by Jácome and Marques ${ }^{21}$ involved four databases and limited search terms, included only one RCT, and did not provide any effect estimates or meta-analyses.
The prevalence of COPD in Denmark is among the highest in the world. ${ }^{24,25}$ Therefore, the health-care-related burden of COPD is considerable. The socioeconomic consequences of offering PR to all patients with COPD have, to our knowledge, not been explored, and this was not within the scope of our study. However, we decided on a weak recommendation of routine PR in COPD with mild symptoms for several reasons:

First, we found moderate quality of evidence for both a statistically and clinically significant improvement in HRQoL immediately after the intervention. The HRQoL was assessed using SGRQ, and as we found an improvement of 4.2 units on the SGRQ scale (0-100 points), this was just within what is defined as clinically relevant ( $>4$-point improvement). ${ }^{16}$ This result was noteworthy since the baseline values for SGRQ were relatively low, suggesting that PR can improve HRQoL in a clinically significant manner in patients with COPD and a relatively preserved health status. However, it is a concern that it was not possible to blind participants when assessing this subjective outcome. In contrast, the aforementioned study of Kruis et al investigated the effects of an integrated COPD management (including exercise) in primary care, but found this intervention to be without effects on the quality of life. The authors state that the low intensity of the exercise training and the unsupervised nature of the program might have contributed to the negative findings, but that they were, nevertheless, representative for a primary care setting. ${ }^{10}$

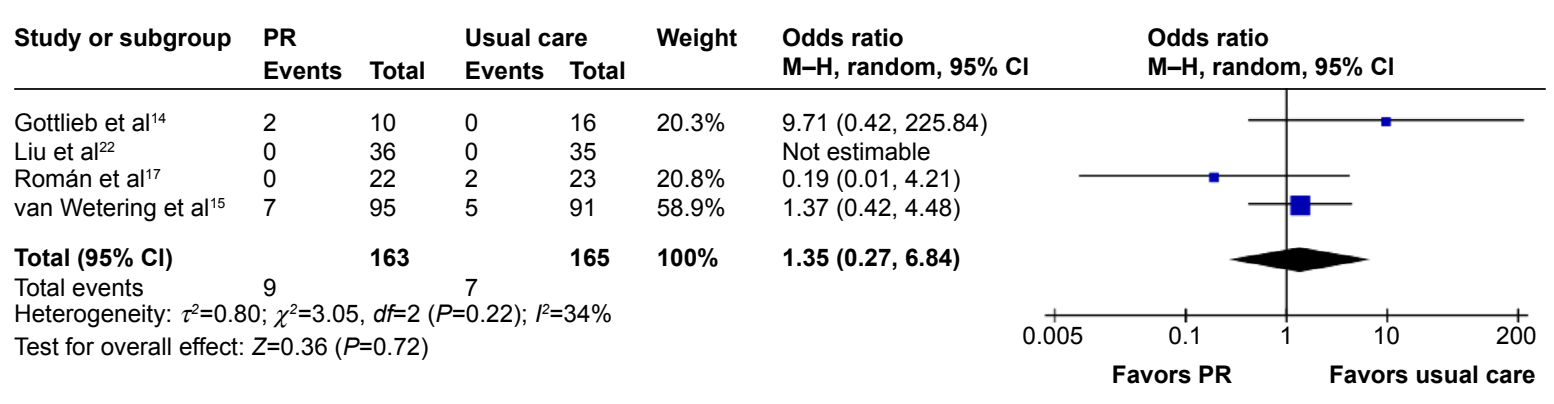

Figure 6 PR versus usual care: mortality, odds ratio.

Abbreviations: $\mathrm{Cl}$, confidence interval; df, degrees of freedom; $\mathrm{M}-\mathrm{H}$, Mantel-Haenszel test; PR, pulmonary rehabilitation; $\square$, point estimate; $\diamond$, pooled effect estimate. 
The authors advise the application of more intensive exercise programs for patients with a higher burden of disease since these patients possess greater potential for improvement. ${ }^{10}$

On the basis of very low quality of evidence, we could not detect beneficial effects of PR on HRQoL when assessed at the longest follow-up. Thus, the observed initial beneficial effects on HRQoL were lost after 18-24 months even though two of the included studies provided maintenance programs for the participants. ${ }^{14,15}$ This result is in agreement with estimates of the long-term effects of PR when provided to more advanced stages of COPD. ${ }^{26}$

Second, no adverse effects with PR were reported in any of the included RCTs, which is in keeping with our clinical experience. ${ }^{14,15,17,22}$ Additionally, we found no difference in total mortality between the PR groups and the control groups, which was also in accordance with our expectations. A significant correlation between physical activity levels and a lower mortality was recently described in a systematic review by Gimeno-Santos et $\mathrm{al}^{7}$, and a recent longitudinal study based on the general population of Copenhagen indicated that low baseline values of physical activity are associated with increased mortality. ${ }^{6}$ Furthermore, determining whether the observed association between physical activity and mortality in the studies was causal or consequential is difficult, and in order to determine the effects of long-term adherence to physical activity on mortality, prospective studies are needed. However, when studying a relatively asymptomatic subgroup of COPD patients, it is difficult to determine the effects of PR on mortality, since the follow-up is relatively short and the number of participants is small.

Third, our results suggest that PR slightly improves exercise capacity, indicated by a statistically significant improvement of the 6MWD in patients with COPD and mild symptoms. However, the average improvement of $26 \mathrm{~m}$ is well below what is considered the minimal clinically relevant improvement, and it is unknown whether the conventional outcomes for evaluating the impact of $\mathrm{PR}$ are applicable to these mostly asymptomatic patients. Thus, whether the effects of PR can be assessed properly using the 6MWD has previously been questioned, ${ }^{1,27}$ since a "ceiling effect" has been demonstrated in patients with COPD and mild symptoms, which obviously limits the ability of the test to detect improvements in exercise capacity. ${ }^{27}$ The use of cycle endurance testing as another measure of exercise capacity was suggested by Laviolette et $\mathrm{al}^{23}$ since their study indicated that cycle endurance is more responsive to the improvement of physical capacity after PR than is the 6MWT. Cycle endurance was investigated in only one of the included studies in our review, and thus, not meta-analyzed. However, results from this trial suggested a clinically significant improvement in the PR group. ${ }^{15}$ Further, this study was the only one to assess muscle strength, showing that hand grip strength was significantly improved following PR compared to usual care. ${ }^{15}$

Finally, although positive effects of PR were found, the overall very low quality of evidence led to a weak recommendation. Therefore, clinicians should assess the need for PR individually according to patients' preferences and clinical manifestations of the disease. We suggest that clinical characteristics such as muscle weakness and inactivity should be taken into consideration when referring patients with less advanced COPD to PR, as we would expect greater individual benefits in these patients than the average improvements seen in our analysis.

\section{Limitations}

An important limitation of this study is the high risk of performance bias in the four included studies, in which the lack of blinding of participants and study staff is a recurring issue. ${ }^{14,15,17,22}$ Especially regarding SGRQ, the risk of performance bias must be considered, since this outcome is easily affected by a lack of blinding. Three of the studies had a high risk of attrition bias due to large dropout rates, and consequently, incomplete outcome data. ${ }^{14,15,17}$

Moreover, the high dropout rates reflect a general challenge in PR studies, in which dropout rates vary between $20 \%$ and $40 \%$ in most studies. ${ }^{28}$ In studies of physical exercise, it is very likely that patients with the poorest health status would drop out more frequently in the intervention arm, leading to an effect estimate biased in favor of the intervention, but this would likely not lead to differential attrition. ${ }^{29}$

For HRQoL, it must be considered that participants who experience deterioration or no change during the studies are at a higher risk of dropping out, which could also skew results in favor of the intervention.

As with any review, a limitation of this study is the comparability of the intervention, setting, duration of the trial, etc, between the studies. Finally, we did not find sufficient data to produce meta-analyses for muscle strength and the maximal exercise capacity.

\section{Conclusion}

On the basis of moderate quality of evidence, we found a small, clinically and statistically significant effect in favor of PR on HRQoL when assessed immediately after the intervention in patients with COPD and mild symptoms. For HRQoL 
assessed 18-24 months after baseline, no improvement was seen, based on very low quality of evidence.

Further, we found low-to-moderate-quality evidence of improved exercise capacity indicated by increased cycle endurance and 6MWD, but the effect on 6MWD was not regarded as clinically relevant. An overall very low quality of evidence and these moderately positive results on shortterm HRQoL and exercise capacity led to a weak recommendation for the use of routine PR in patients with COPD and mild symptoms.

\section{Acknowledgments}

We thank Gottlieb et al, Román et al and van Wetering et al who all kindly provided us additional data regarding their studies. A special thanks to both Conni Skrubbeltrang, who assisted in performing the literature search, and Professor Jørgen Vestbo, MD, DMsc, who contributed with valuable comments.

The Centre of Inflammation and Metabolism (CIM) is supported by a grant from the Danish National Research Foundation (DNRF55). The Centre for Physical Activity Research (CFAS) is supported by a grant from Trygfonden. This study was further supported by the Danish Health and Medicines Authority, the Axel Muusfeldt Foundation, the Capital Region of Denmark, and the Novo Nordisk Foundation. CIM is a member of DD2 - the Danish Center for Strategic Research in type 2 diabetes (the Danish Council for Strategic Research, grant no 09-067009 and 09-075724).

\section{Disclosure}

PL has received research grants from Boehringer Ingelheim, GlaxoSmithKline, Novartis, and Pfizer; received fee for speaking from AstraZeneca, Boehringer Ingelheim, GlaxoSmithKline, Novartis, Nycomed, Pfizer, and Sandoz; and received fee for consulting from Almirall, AstraZeneca, Boehringer Ingelheim, GlaxoSmithKline, Mundipharma, Novartis, Nycomed, Sandoz, and Pfizer. All the authors report no other conflicts of interest in this work.

\section{References}

1. Lacasse Y, Martin S, Lasserson TJ, Goldstein RS. Meta-analysis of respiratory rehabilitation in chronic obstructive pulmonary disease. A Cochrane systematic review. Eur Medicophys. 2007;43(4):475-485.

2. Bolton CE, Bevan-Smith EF, Blakey JD, et al. British Thoracic Society guideline on pulmonary rehabilitation in adults. Thorax. 2013;68(Supp12): ii1-ii30.

3. Marciniuk DD, Brooks D, Butcher S, et al. Optimizing pulmonary rehabilitation in chronic obstructive pulmonary disease - practical issues: a Canadian Thoracic Society Clinical Practice Guideline. Can Respir J. 2010;17(4):159-168.
4. Spruit MA, Singh SJ, Garvey C, et al. An official American Thoracic Society/European Respiratory Society statement: key concepts and advances in pulmonary rehabilitation. Am J Respir Crit Care Med. 2013;188(8):e13-e64.

5. The Danish Health and Medicines Authority. KOL - Kronisk obstruktiv lungesygdom: Anbefalinger for tidlig opsporing, opfølgning, behandling og rehabilitering [COPD - chronic obstructive pulmonary disease. Recommendations for early recognition, follow-up, treatment and rehabilitation]. Version 2; 2007. Available from: http://sundhedsstyrelsen. $\mathrm{dk} / \mathrm{da} /$ sundhed/puljer-og-projekter/projekter/kol-projektet-tidligopsporing,-opfoelgning,-behandling-og-rehabilitering-af-kol/ kol-anbefalinger-for-tidlig-opsporing,-opfoelgning,-behandling-ogrehabilitering. Accessed September 1, 2014. Danish.

6. Vaes AW, Garcia-Aymerich J, Marott JL, et al. Changes in physical activity and all-cause mortality in COPD. Eur Respir J. 2014;44(5): 1199-1209.

7. Gimeno-Santos E, Frei A, Steurer-Stey C, et al. Determinants and outcomes of physical activity in patients with COPD: a systematic review. Thorax. 2014;69(8):731-739.

8. Troosters T, van der Molen T, Polkey M, et al. Improving physical activity in COPD: towards a new paradigm. Respir Res. 2013;14(1):115.

9. Garcia-Aymerich J, Serra I, Gomez FP, et al. Physical activity and clinical and functional status in COPD. Chest. 2009;136(1):62-70.

10. Kruis AL, Boland MR, Assendelft WJ, et al. Effectiveness of integrated disease management for primary care chronic obstructive pulmonary disease patients: results of cluster randomised trial. $B M J$. 2014;349:g5392.

11. The Grading of Recommendations Assessment Development and Evaluation (GRADE) Working Group. Available from: http://www. gradeworkinggroup.org/. Accessed August 8, 2014.

12. The Danish Health and Medicines Authority. Nationale Kliniske Retningslinjer [National Clinical Guidelines]. Available from: https:// sundhedsstyrelsen.dk/da/sundhed/kvalitet-og-retningslinjer/nationalekliniske-retningslinjer. Updated August 21, 2014. Accessed August 29, 2014. Danish.

13. Guyatt GH, Oxman AD, Kunz R, et al. GRADE guidelines, 2: framing the question and deciding on important outcomes. $J$ Clin Epidemiol. 2011;64(4):395-400.

14. Gottlieb V, Lyngso AM, Nybo B, Frolich A, Backer V. Pulmonary rehabilitation for moderate COPD (GOLD 2) - does it have an effect? COPD. 2011;8(5):380-386.

15. van Wetering CR, Hoogendoorn M, Mol SJ, Rutten-van Molken MP, Schols AM. Short- and long-term efficacy of a community-based COPD management programme in less advanced COPD: a randomised controlled trial. Thorax. 2010;65(1):7-13.

16. Jones PW. Interpreting thresholds for a clinically significant change in health status in asthma and COPD. Eur Respir J. 2002;19(3): 398-404.

17. Román M, Larraz C, Gomez A, et al. Efficacy of pulmonary rehabilitation in patients with moderate chronic obstructive pulmonary disease: a randomized controlled trial. BMC Fam Pract. 2013;14:21.

18. Redelmeier DA, Bayoumi AM, Goldstein RS, Guyatt GH. Interpreting small differences in functional status: the Six Minute Walk test in chronic lung disease patients. Am J Respir Crit Care Med. 1997;155(4): $1278-1282$.

19. The Cochrane Collaboration. Cochrane Handbook for Systematic Reviews of Interventions, Version 5.1.0. Available from: www. cochrane-handbook.org. Updated March 2011. Accessed August 11, 2014.

20. Review Manager 5.3 [computer program]. Copenhagen, Denmark: The Nordic Cochrane Centre, The Cochrane Collaboration; 2014.

21. Jácome C, Marques A. Pulmonary rehabilitation for mild COPD: a systematic review. Respir Care. 2014;59(4):588-594.

22. Liu X-D, Jin H-Z, Ng BH-P, Gu Y-H, Wu Y-C, Lu G. Therapeutic effects of qigong in patients with COPD: a randomized controlled trial. HKJOT. 2012;22(1):38-46. 
23. Laviolette L, Bourbeau J, Bernard S, et al. Assessing the impact of pulmonary rehabilitation on functional status in COPD. Thorax. 2008; 63(2):115-121.

24. Buist AS, McBurnie MA, Vollmer WM, et al. International variation in the prevalence of COPD (The BOLD Study): a population-based prevalence study. Lancet. 2007;370(9589):741-750.

25. Fabricius P, Løkke A, Marott JL, Vestbo J, Lange P. Prevalence of COPD in Copenhagen. Respir Med. 2011;105(3):410-417.

26. Beauchamp MK, Evans R, Janaudis-Ferreira T, Goldstein RS, Brooks D. Systematic review of supervised exercise programs after pulmonary rehabilitation in individuals with COPD. Chest. 2013; 144(4):1091-1093.
27. Smith DR. Use of the 6-Min Walk Test: A Pro and Con Review. PCCSU article. 23, 2009 June. Available from: http://69.36.35.38/accp/ pccsu/use-6-min-walk-test-pro-and-con-review?page=0,3. Accessed September 1, 2014.

28. Fischer MJ, Scharloo M, Abbink JJ, et al. Drop-out and attendance in pulmonary rehabilitation: the role of clinical and psychosocial variables. Respir Med. 2009;103(10):1564-1571.

29. Almadana V, Romero C, Valido A, Gómez-Bastero A, Sánchez J, Montemayor T. Profile of patients who drop out of a pulmonary rehabilitation program [meeting abstracts]. Chest. 2014;145(Suppl 3):370A.

International Journal of COPD

\section{Publish your work in this journal}

The International Journal of COPD is an international, peer-reviewed journal of therapeutics and pharmacology focusing on concise rapid reporting of clinical studies and reviews in COPD. Special focus is given to the pathophysiological processes underlying the disease, intervention programs, patient focused education, and self management protocols.

\section{Dovepress}

This journal is indexed on PubMed Central, MedLine and CAS. The manuscript management system is completely online and includes a very quick and fair peer-review system, which is all easy to use. Visit $\mathrm{http}: / / \mathrm{ww}$.dovepress.com/testimonials.php to read real quotes from published authors.

Submit your manuscript here: http://www.dovepress.com/international-journal-of-chronic-obstructive-pulmonary-disease-journal 